\title{
Advances in Modern Capacitive ECG Systems for Continuous Cardiovascular Monitoring
}

\author{
A. Schommartz, B. Eilebrecht, T. Wartzek, M. Walter, S. Leonhardt
}

\begin{abstract}
The technique of capacitive electrocardiography (cECG) is very promising in a flexible manner. Already integrated into several everyday objects, the single lead cECG system has shown that easy-to-use measurements of electrocardiograms are possible without difficult preparation of the patients. Multi-channel cECG systems enable the extraction of ECG signals even in the presence of coupled interferences, due to the additional redundant information. Thus, this paper presents challenges for electronic hardware design to build on developments in recent years, going from the one-lead cECG system to multi-channel systems in order to provide robust measurements - e.g. even while driving an automobile.
\end{abstract}

Keywords: capacitive electrodes, non-contact measurements, ECG, multi-channel sensor array, automotive application.

\section{Introduction}

Cardiovascular diseases have for years been the most common cause of death (German Federal Statistical Office). Today, however, it is essential to monitor cardiac patients not only in hospitals, but also in everyday life (e.g. while driving a car), because of the demographic change toward an aging population. In traffic situations, we do not risk only our own lives, and this makes monitoring even more important.

The electrocardiogram (ECG) has been known for a long time as a fast examination tool which can provide important clues to the status of the cardiovascular system. It is often the tool of first choice in emergency situations, e.g. for detecting a heart attack. However, until now no extensive long-time monitoring system for people in high-risk groups could be developed, for technical and financial reasons.

In recent years, a technique already known since 1967 through Richardson has been in the main research focus: measuring potentials with isolated electrodes [1]. This capacitive measurement method is nowadays built into a range of everyday objects: office chairs [2,3], bathtubs [4], toilet seats [5], incubators $[6]$, cars $[7,8]$ and beds [9]. As an example, the socalled "Aachen SmartChair" from [10] integrates two capacitive electrodes in an off-the-shelf office chair (see Figure 1) for easy-to-use measurements where no medical staff is needed and no difficult preparation has to be done.

Even a study on acceptability for medical staff and patients produced very positive results [11]. However, this technique has not yet been marketed, perhaps because most systems use a single lead, which is less robust than conventional conductive systems.

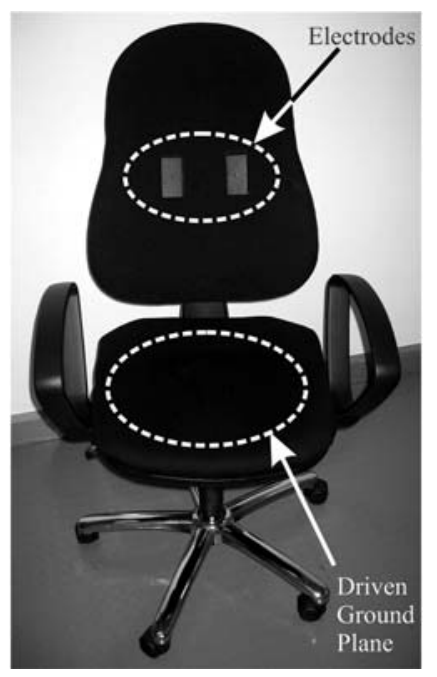

Fig. 1: The "Aachen SmartChair": A single lead cECG system in an office chair [10]

This paper presents an overview of the capacitive measurement method, the challenges for electronic hardware design and the developments in recent years from single lead systems to multi-channel systems for robust and reliable measurements.

\section{Theory of capacitive ECG measurements}

Charge movements on the surface of human bodies, caused by their heart activity, influence the electric charge distribution on an electrode within a small distance from the body. This knowledge is used for capacitive measurements.

Conductive electrodes, often made of $\mathrm{Ag} / \mathrm{AgCl}$, need a contact gel as a conductive contact to the 
body surface to measure the potential between the two electrodes. This is mainly resistive behavior. With time, the contact gel dehydrates and reduces the quality of the signals.

Capacitive electrodes, isolated e.g. by a very thin lacquer coating with high surface resistance, need no leading contact to the body. In addition, the potentials can be measured even through several layers of clothing (depending on material and thickness). Each electrode forms a coupling capacitance $C$ with the patient's body, which is known to be

$$
C=\epsilon_{0} \epsilon_{r} \frac{A}{d}
$$

where $A$ is the effective surface area of the electrode, $d$ is the thickness, $\epsilon_{r}$ is the dielectric constant of the clothes, and $\epsilon_{0}$ is vacuum permittivity. With $d=0.3 \mathrm{~mm}$ (off-the-shelf cotton shirt), $\epsilon_{r}=1$ and $A=40 \mathrm{~mm} \times 80 \mathrm{~mm}$, as in [10], the coupling capacitance is about $90 \mathrm{pF}$. For a high coupling capacitance, large electrode surfaces within small distances from the body are essential.

One major disadvantage of this technique is the continuous changing of $C$ due to patients' movements, transpiration, varying thickness $d$ and clothing materials, as well as static charges. In addition, it is a technical challenge to deal with high impedance biosignals in the range of a few $\mathrm{mV}$. Direct impedance conversion must be achieved in order to avoid disturbing voltages.

\section{Realization of capacitive ECG}

The coupling capacitance forms the input of a high impedance input stage for impedance transformation (Figure 2). Typically, a high-impedance bias resistor $R_{\text {Bias }}(>G \Omega)$ is connected to ground to discharge to capacitance, but this results in a first order high pass behavior with the corresponding cut-off frequency:

$$
f_{c}=\frac{1}{2 \pi R_{\mathrm{BiaS}} C}
$$

Thus, variations of the coupling capacitances can result in a shift of $0.1-100 \mathrm{~Hz}$ of the cut off frequency into the spectrum of the ECG, and can thus deteriorate the ECG signal.

Even the operational amplifier should have a very high input impedance for the same reason. Further on, due to the small voltage amplitudes, low noise amplifiers should be used and because of the shift of the operating point low bias currents are necessary.

Signals measured at two different positions are combined, forming the differential input in an instrumentation amplifier with a high CMRR (common mode rejection ratio) of about $120 \mathrm{~dB}$ in order to fil-

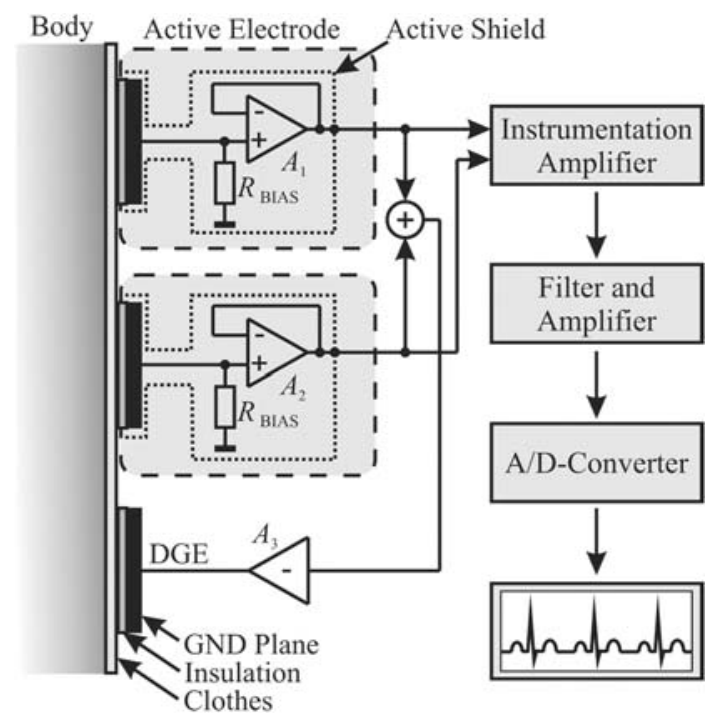

Fig. 2: Block diagram of the capacitive ECG system, modified from [10]

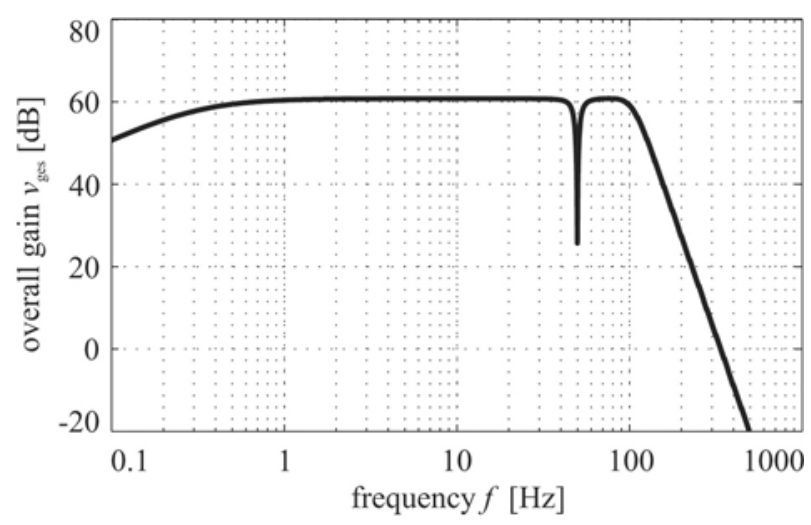

Fig. 3: Matlab simulation of the resulting gain in the spectrum of the ECG after filtering

ter the coupled power line interferences that underly both signals. Due to the asymmetries, e.g. variable length of wires or varying distances of electrodes, coupled interference may have a slightly different effect. Therefore it cannot be eliminated in reality as well as in theory. Further filtering of the signal is indispensable.

A low pass filter is used to cut off the high frequency components (typically $>100 \mathrm{~Hz}$ ), which do not provide information for the ECG, as well as higher harmonics of the $50 \mathrm{~Hz}$ power baseline. With an additional notch filter at $50 \mathrm{~Hz}$ this noise can be suppressed. A high pass filter with a low cut off frequency of about $0.3 \mathrm{~Hz}$ deletes the DC components and minimizes the baseline drifts. Figure 3 shows the Matlab simulation of the filter and gain cascade, depending on the frequency, in the range of $0.1-1000 \mathrm{~Hz}$. A nearly constant gain of $61 \mathrm{~dB}$ in the useful frequency range was achieved. The high pass filter with a cut off frequency of $0.3 \mathrm{~Hz}$ shows 
moderate absorption, whereas the high pass of the capacitive electrodes (due to uncertainties of the modelling depending on the distance between electrode and body) is not considered in this simulation. The notch filter at $50 \mathrm{~Hz}$ achieves suppression of $35 \mathrm{~dB}$ with regard to the desired signal. The remaining gain of $26 \mathrm{~dB}$ referring to the input signal is low enough. Higher suppression can be achieved by additional notch filter stages or digital signal processing.

Controlling further components of the signal processing of the ECG signal, i.e. the A/D converter, significant amplification (about factor 1000) is usually implied at good SNR (signal to noise ratio). First amplification can be realized by external circuitry of the instrumentational amplifier increasing the SNR without driving the subsequent components into saturation. The main amplification takes place at the end of the filter cascade in the output or gain stage. Figure 4 shows a realization of an ECG circuit with two simultaneous filter cascades.

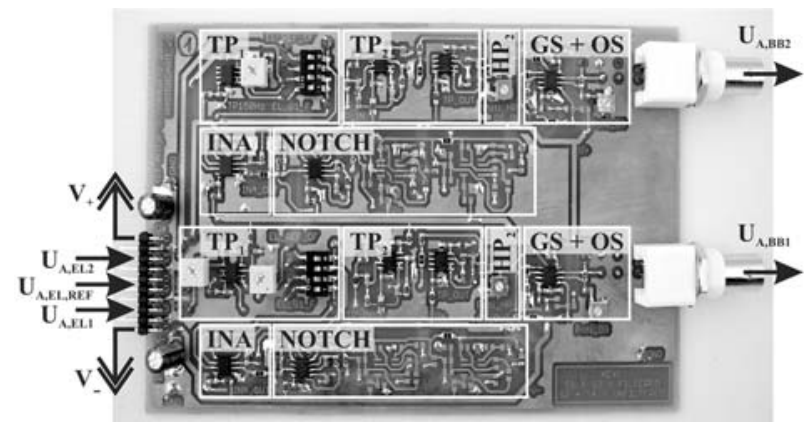

Fig. 4: Realization of an ECG circuit for two simultanous measurements of ECG

Conventional ECG measurement systems make use of the principle knwon as "Driven Right Leg" to eliminate interference in the signal even more $[12,13]$. This principle can also achieve interference reduction in capacitive ECG measurements. There it is often referred to as "Driven Ground Plane", as in [7], or "Driven Ground Electrode" (DGE), as in [14]. The negative feedback electrode connects the body and the output of an inverting operational amplifier the potential of which is the sum of the active electrodes, with amplification of about -1000 . In this way, identical potential changes on the body surface are not transferred to the system output (compare [7]).

Dealing with these technical challenges alone will not ensure a robust cECG measurement system. Challenges due to the patient also need to be overcome.

\section{Results}

Progress in digital circuit technology in recent years has enabled the implementation of complex algorithms in reasonable modules. Improved types of electrodes integrated in everyday objects have been achieved and there have been advances in signal processing.
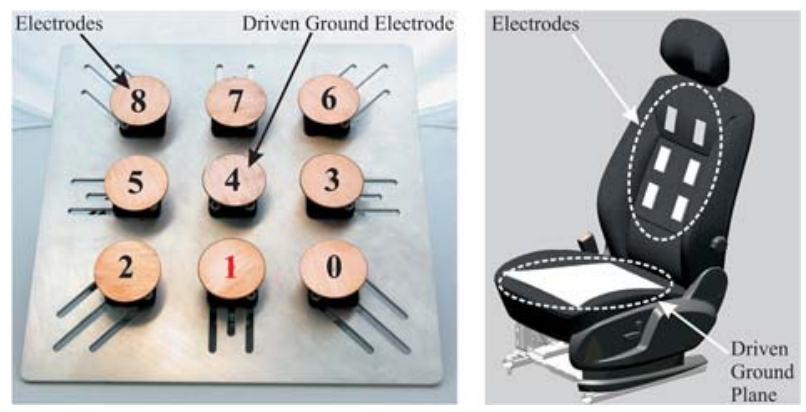

Fig. 5: Left: Experimental setup for multi-channel cECG, right: Multi-channel cECG integrated into the driver's seat of a Ford automobile

The latest $\mathrm{cECG}$ systems have been realized for multi-channel measurements. This redundancy may lead to robust measurements to ensure reliable medical statements on the basis of an ECG. As a first outcome, a multi-channel cECG system has been presented by [15], with 15 electrodes integrated in a tablet pc. However, the patient must additionally be connected to ground. To the best of the author's knowledge, our group presented the first independent multi-channel cECG system with free choice for the negative feedback DGE to reduce the common mode rejection [14]: on a square aluminum plate an array of nine round electrodes, positioned in a $3 \times 3$ matrix, was attached with flanged bearings enabling to tilt every electrode (Figure 5 left). In combination with the ability to have continuously adjustable positions of the electrodes on the plate, this allows proper adaptation to the silhouette of the patients. Ancillary connectors with springs have been developed to achieve proper adaptation and surface pressure in axial direction.

This construction allows simultaneous measurements of up to eight cECGs where the leads can be chosen manually, just like the DGE.

Figure 6 shows a sequence of six leads according to Einthoven (3) and Goldberger (4), measured on the patient's back, which can be calculated as:

$$
\begin{aligned}
\mathrm{I} & =U_{8}-U_{6}=\phi_{8}-\phi_{6}, \\
\mathrm{II} & =U_{8}=\phi_{8}-\phi_{1}, \\
\mathrm{III} & =U_{6}=\phi_{6}-\phi_{1}, \\
\mathrm{aVR} & =U_{8}-\frac{U_{0}+U_{6}}{2}, \\
\mathrm{aVL} & =U_{6}-\frac{U_{0}+U_{8}}{2}, \\
\mathrm{aVF} & =U_{0}-\frac{U_{6}+U_{8}}{2},
\end{aligned}
$$


where $\phi_{i}$ is the potential from the electrode $i, \phi_{1}$ is the reference potential and the voltage $U_{i}=\phi_{i}-\phi_{1}$ (compare Figure 5 left). The sequence in Figure 6 shows that every channel has robust signals and the R-peaks can be clearly identified and therefore used e.g. for QRS detection.

The possibility of developing a multi-channel cECG system enables integration into the driver's seat (here a Ford S-Max) for cardiovascular monitoring even in traffic situations (Figure 5 right). The positions of the electrodes in a $2 \times 3$ matrix in the back rest were chosen due to pressure measurements with a flexible pressure sensor mattress by a group of ten males and females with different physique. Averaging the pressure distribution identified the best positions, and these were then verified by cECG measurements. A textile negative feedback electrode integrated into the seat panel is used for common mode reduction.

Measurements of the pressure sensor mattress, with corresponding ECG measurements, the best capacitive channel and reference ECG as a gold standard, for two persons, see Figure 7, show that a proper pressure contact to the electrodes is of essential interest. The BMI, a criterion for the relation between weight and height of human beings, of the male proband in the upper part is normal with $22.2 \mathrm{~kg} / \mathrm{m}^{2}$, and in the lower part the BMI is low with $16.8 \mathrm{~kg} / \mathrm{m}^{2}$. It can be seen that with decreasing surface pressure, the signal quality of the cECG decreased in comparison with the reference ECG below.

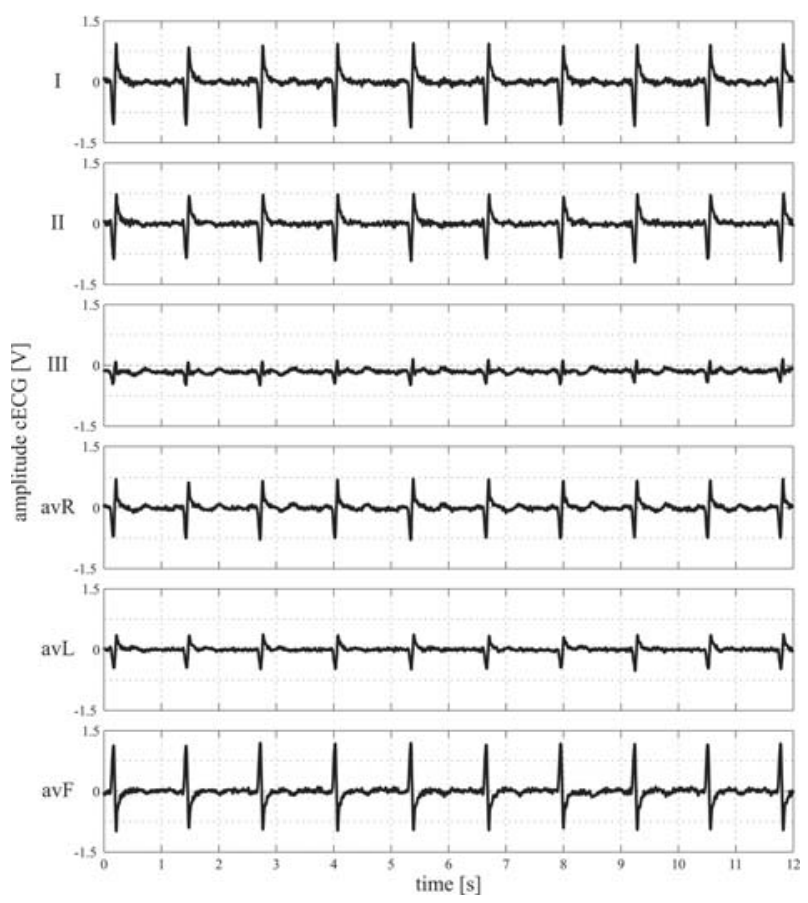

Fig. 6: Sequence of resulting leads according to Einthoven and Goldberger, as shown in (3), (4), transformed to the patient's back
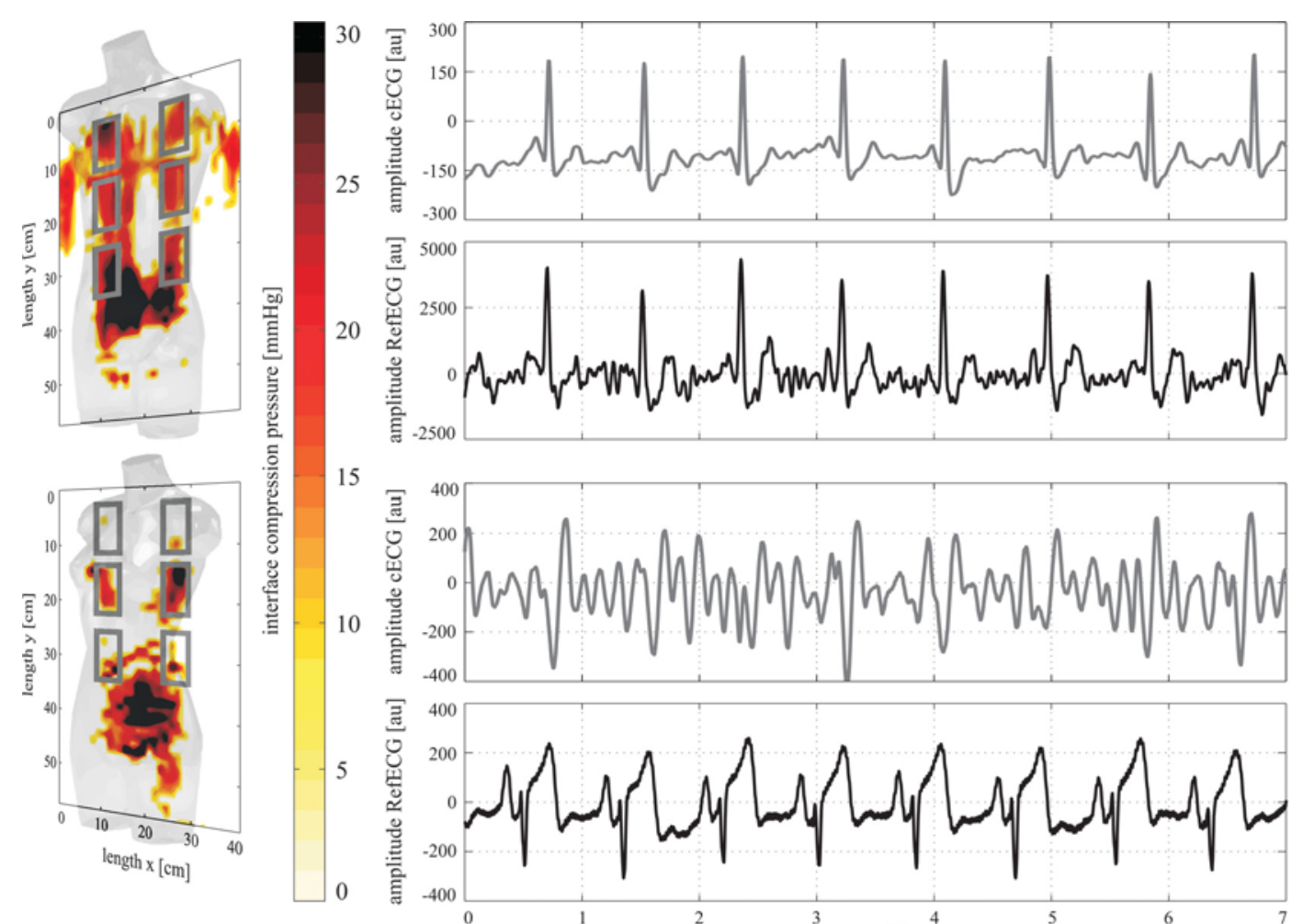

s

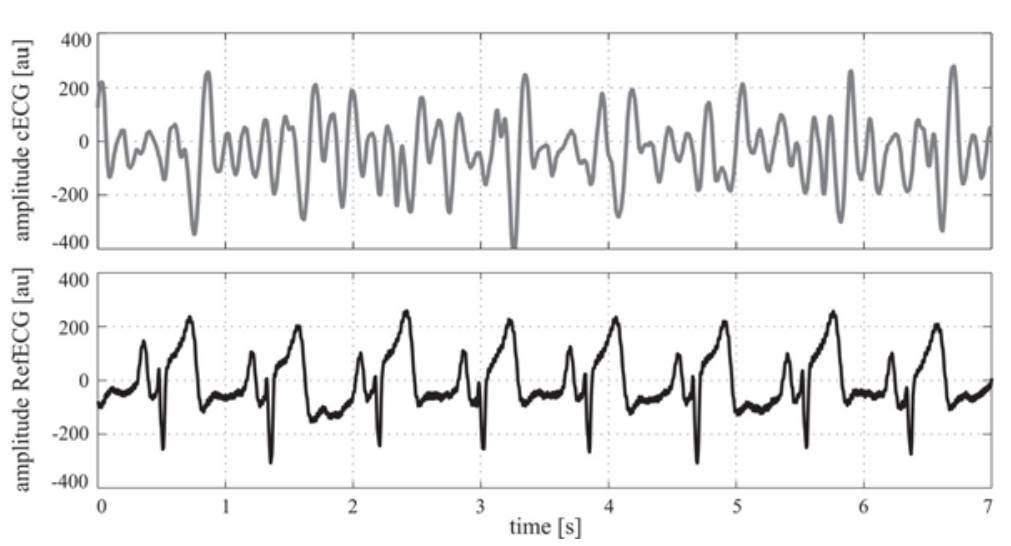

Fig. 7: Pressure measurements of two subjects with different BMI: Male proband with normal BMI in the upper part, below female proband with low BMI. On the right side the EECG and the reference ECG as gold standard are shown for these persons 
In test runs at a Ford car test site, the system was validated in static tests and while driving on different track surfaces. With $93 \%$ of the 59 probands, robust cECGs were measured in static tests. On motorway tracks, in particular, the detection rate of $92.4 \%$ is very robust [16]. City tracks, with more steering motion and road damage, lead to lower detection rates than for the reference ECG. Importantly, irregularities in the ECG could be detected without restricting the driver's level of comfort.

\section{Conclusion}

In recent years, capacitive ECG measurement systems developed by various groups have shown that this technique is very flexible due to integration into several everyday objects. However, the medical diagnostics and the robustness of the systems are limited because most systems are based on a single lead. Multi-channel cECG systems present a promising approach for monitoring people in high-risk groups. Even measurements while driving a car on a test track showed good performances. This is a major step towards monitoring and assisting drivers.

\section{Acknowledgement}

Parts of the research described in this paper have received funding from the European Community's Seventh Framework Programme under grant agreement No. FP7-216695 and the Ford Forschungszentrum Aachen GmbH, Aachen, Germany.

\section{References}

[1] Richardson, P.: The insulated electrode. Proceedings of the 20th Annual Conference on Engineering in Medicine and Biology. Boston, 1967, vol. 157.

[2] Lim, Y., Kim, K., Park, S.: ECG measurement on a chair without conductive contact. IEEE Transactions on Biomedical Engineering, 2006, vol. 53, no. 5 , p. $956-959$.

[3] Aleksandrowicz, A., Walter, M., Leonhardt, S.: Ein kabelfreies, kapazitiv gekoppeltes EKGMesssystem/Wireless ECG measurement system with capacitive coupling. Biomedizinische Technik, 2007, vol. 52, no. 2, p. 185-192.

[4] Lim, Y., Kim, K., Park, K.: The ECG measurement in the bathtub using the insulated electrodes. 26th Annual International Conference of the IEEE EMBS, 2004, vol. 1.

[5] Kim, K., Lim, Y., Park, K.: The electrically noncontacting ECG measurement on the toilet seat using the capacitively-coupled insulated electrodes. 26th Annual International Conference of the IEEE EMBS, 2004, vol. 1.

[6] Kato, T., Ueno, A., Kataoka, S., Hoshino, H., Ishiyama, Y.: An application of capacitive electrode for detecting electrocardiogram of neonates and infants. 28th Annual International Conference of the IEEE EMBS, 2006, p. 916-919.

[7] Leonhardt, S., Aleksandrowicz, A.: Non-contact ECG monitoring for automotive application. 5th International Summer School and Symposium on Medical Devices and Biosensors, 2008, p. $183-185$.

[8] Chamadiya, B., Heuer, S., Hofmann, U., Wagner, M.: Towards a capacitively coupled electrocardiography system for car seat integration. In Proceedings IFMBE, 2008, vol. 22, p. 1217-1221.

[9] Ishijima, M.: Monitoring of electrocardiograms in bed without utilizing body surface electrodes. IEEE Transactions on Biomedical Engineering, 1993, vol. 40, no. 6, p. 593-594.

[10] Aleksandrowicz, A., Leonhardt, S.: Wireless and non-contact ECG measurement system the Aachen SmartChair. Acta Polytechnica, 2007, vol. 2, p. 68-71.

[11] Czaplik, M., Eilebrecht, B., Ntouba, A., Walter, M., Schauerte, P., Leonhardt, S., Rossaint, R.: Clinical proof of practicability for an ECG device without any conductive contact, Biomedizinische Technik, 2010, vol. 55, p. 291-300.

[12] Neuman, M.: Biopotential amplifiers, Medical Instrumentation: Application and Design, 1978, p. 292-296.

[13] Winter, B., Webster, J.: Driven-right-leg circuit design, IEEE Transactions on Biomedical Engineering, 1983, p. 62-66.

[14] Eilebrecht, B., Schommartz, A., Walter, M., Wartzek, T., Czaplik, M., Leonhardt, S.: A capacitive ECG array with visual patient feedback, 32nd Annual International Conference of the IEEE EMBS, 2010.

[15] Oehler, M., Ling, V., Melhorn, K., Schilling, M.: A portable ECG system with capacitive sensors, Physiological Measurement, 2008, vol. 29, p. $783-793$.

[16] Eilebrecht, B., Wartzek, T., Lem, J., Vogt, R., Leonhardt, S.: Capacitive electrocardiogram measurement system in the driver seat, Automobiltechnische Zeitschrift ATZ, 2011, vol. 113, p. $50-55$. 


\section{About the authors}

Antje Schommartz was born in Essen, Germany, on March 31st, 1981. She studied Electrical Engineering and Information Technology, specializing in Medical Engineering, at Ruhr University Bochum, Germany, where she received her Dipl.-Ing. degree in 2009. She currently works as a Ph.D. student at the Philips Chair of Medical Information Technology, RWTH Aachen University, Germany. Her research interests are focused on capacitive ECG measurements and high frequency cardiac neuromodulation. She is a member of the VDE, DGBMT and IEEE.

Benjamin Eilebrecht was born in Bochum, Germany, on June 6th, 1982. He studied Electrical Engineering, with a specialization of Medical Engineering, at Ruhr University Bochum, Germany, and received his Dipl.-Ing. degree in 2008. He is working as a Ph.D. candidate at the Philips Chair of Medical Information Technology, RWTH Aachen University, Aachen, Germany. His research interests include non-contact monitoring techniques, learning algorithms and modeling. He has been a member of the German Electrical Engineering Association (VDE) since December 2008.

Tobias Wartzek was born in Krefeld in 1982. From 2003 to 2008, he studied electrical engineering focusing on information and communication technology, and received his Dipl.-Ing. degree from RWTH Aachen University, Germany in 2008. He is currently pursuing a Ph.D. degree at the Chair of Medical Information Technology, RWTH Aachen University, Aachen, Germany, where he is also working as a research assistant. His research interests are in the field of modeling physiological systems, new sensors for biomedical measurements, and automation and diagnosis support for the intensive care unit.
Marian Walter was born in Saarbrücken, Germany, on March 4th, 1966. He studied Electrial Engineering, with a specialization in Control Engineering, at the Technical University of Darmstadt and received his Dipl.-Ing. degree in 1995 and his Dr.-Ing. degree in 2002. He has worked in the medical engineering industry for three years and was appointed senior scientist and deputy head at the Philips Chair of Medical Information Technology at RWTH Aachen University, Aachen, Germany, in 2004. His research interests include non-contact monitoring techniques, signal processing and feedback control in medicine.

Steffen Leonhardt was born in Frankfurt, Germany, on Nov. 6th, 1961. He holds an M.S. in Computer Engineering from SUNY at Buffalo, NY, USA, a Dipl.-Ing. in Electrical Engineering and a Dr.Ing. degree in Control Engineering from the Technical University of Darmstadt, Germany, and a M.D. in Medicine from J. W. Goethe University, Frankfurt, Germany. He has 5 years of R\&D management experience in medical engineering industry and was appointed Full Professor and Head of the Philips endowed Chair of Medical Information Technology at RWTH Aachen University, Germany in 2003. His research interests include physiological measurement techniques, personal health care systems and feedback control systems in medicine.

Antje Schommartz

E-mail: schommartz@hia.rwth-aachen.de

Benjamin Eilebrecht

Tobias Wartzek

Marian Walter

Steffen Leonhardt

Philips Chair for Medical Information Technology

RWTH Aachen University

Pauwelsstr. 20, 52074 Aachen, Germany 